\title{
Using terlipressin in a pediatric patient with septic shock resistant to catecholamines
}

\author{
Seher Erdogan, Mehmet Bosnak \\ Department of Pediatric Critical Care, Gaziantep University, Gaziantep, Turkey
}

\begin{abstract}
Sepsis and septic shock are important causes of morbidity and mortality in critically ill children. The goal of treatment is to ensure adequate mean arterial pressure to maintain organ perfusion. The growing number of instances of peripheral vascular hyporeactivity to catecholamines has necessitated the search for alternative vasopressors. A 14-year-old boy had septic shock, with a high cardiac index and low systemic vascular resistance index (SVRI) measurements according to pulse contour analysis, despite treatment with dopamine, dobutamine, adrenaline, and noradrenaline infusions. A terlipressin (TP) $10 \mu \mathrm{g} / \mathrm{kg}$ intravenous bolus was administered, followed by a 1 $\mu \mathrm{g} / \mathrm{kg} / \mathrm{minute}$ continuous infusion. The response to TP treatment was assessed using pulse contour analysis. The mean arterial pressure and SVRI increased, and the cardiac index and heart rate decreased within 10 minutes after bolus administration of TP. Noradrenaline infusion could be reduced to $0.7 \mu \mathrm{g} / \mathrm{kg} /$ minute within 5 hours. The goal in presenting this case was to evaluate the vasoconstrictor effects of TP, a long-acting vasopressin analogue, in septic shock.
\end{abstract}

Keywords: Child; septic shock; terlipressin.

S epsis and septic shock are important causes of morbidity and mortality in critically ill pediatric cases [1]. Septic shock is a form of distributive shock characterized by arterial and venous vasodilation. The target in the treatment is to achieve a mean arterial pressure that will maintain sufficient organ perfusion. Vascular hyporeactivity to catecholamines has compelled the search for an alternative vasopressor agent. Terlipressin (TP) is a long-acting synthetic analogue of vasopressin with an affinity for vascular receptors greater than that of vasopressin. TP has also been demonstrated to be more effective than noradrenaline in patients with noradrenaline-resistant septic shock.

Hemodynamic monitoring with a pulse-induced contour cardiac output (PiCCO) monitor (Pulsion Medical Systems SE, Feldkirchen, Germany) can continuously measure cardiac output at the femoral artery without the need for pulmonary artery intervention. In addition, it can assess cardiac preload and extravascular lung water (EVLW). Therefore, use of a pulse contour method is very valuable in the monitoring of fluid balance, especially in patients under mechanical ventilation. 
Presently described is the case of a 14-year-old boy who had catecholamine-resistant septic shock and underwent TP treatment. A pulse contour method used to monitor his fluid balance, and the potential use of TP as a treatment alternative are highlighted.

\section{CASE REPORT}

A 14-year-old male patient who had been followed up for 3 years with a diagnosis of Joubert syndrome and chronic renal failure and was receiving peritoneal dialysis treatment was brought into the pediatric emergency service due to vomiting and intolerance to food intake persisting for 1 week. Upon the development of hyponatremia and hypopotassemia, he was hospitalized and the appropriate supplementation treatment was instituted. Blood, urine, and peritoneal fluid cultures were obtained because of febrile episodes. Intravenous (IV) antibiotherapy with tazobactam-ampicillin was initiated. Growth of Candida spp. in his peritoneal culture necessitated the inclusion of liposomal amphotericin B in his treatment, and the peritoneal dialysis catheter was removed. The patient was anuric and had volume overload, so intermittent hemodialysis therapy was initiated and maintained in 3-hour sessions 3 days a week. An abdominal ultrasound was performed, which revealed complicated, lobulated, fluid-filled cystic lesions, the largest with dimensions of nearly $8 \times 5 \mathrm{~cm}$ (pseudocyst) in the pancreatic loge, and multiple cortical cysts in both kidneys. An abdominal computed tomography (CT) scan indicated large cystic lesions localized in the anterior abdominal region that were compressing portions of the gastrointestinal system and intraabdominal organs, and which extended from the subhepatic region to the pelvis. Loculated fluid collections that were connected with each other in the peripancreatic area were noteworthy. The patient was evaluated by the department of pediatric surgery, and surgery was planned. During the operation, a cyst on to the anterior abdominal wall and extending from the pelvis to the epigastric region was observed. During attempts to resect the cyst, which was very strongly adhered to the anterior abdominal wall, the cyst ruptured and nearly $2500 \mathrm{cc}$ serohemorrhagic fluid was aspirated. During the intervention, the patient suffered cardiac arrest. Erythrocyte suspension was transfused, fluid support was provided, and adrenaline and noradrenaline infusions were started as a result of his hypotensive state. The patient was transferred to the intensive care unit. His blood pressure was 55/35 mmHg and his heart rate was $90 \mathrm{bpm}$. His pupils were bilaterally dilated with an absent light reflex. Capillary refill time was 6 to 7 seconds. Other parameters were: arterial blood gas $\mathrm{pH}: 7.15$, $\mathrm{PaCO}_{2} \div 41.8 \mathrm{mmHg}, \mathrm{PaO}_{2} \div 82.8 \mathrm{mmHg}$, base excess: $-13.2 \mathrm{mmol} / \mathrm{L}, \mathrm{HCO}_{3}: 14 \mathrm{mmol} / \mathrm{L}$, lactate: $16 \mathrm{mmol} / \mathrm{L}$, blood urea nitrogen (BUN): $87 \mathrm{mg} /$ $\mathrm{dL}$, and creatinine: $2.7 \mathrm{mg} / \mathrm{dL}$. Mechanical ventilation was provided with the following settings: peak inspiratory pressure: $24 \mathrm{~cm} \mathrm{H} \mathrm{H}_{2} \mathrm{O}$, positive end-expiratory pressure $5 \mathrm{~cm} \mathrm{H}_{2} \mathrm{O}$, frequency: $20 /$ minute, fraction of inspired oxygen: 1.00. Saline solution ( $0.9 \%$ sodium chloride) was given in 2 bolus doses of $20 \mathrm{~mL} / \mathrm{kg}$. Upon persistence of hypotension, dopamine, dobutamine, adrenaline, and noradrenaline infusions were started. The doses of dopamine, and dobutamine were increased to $20 \mu \mathrm{g} / \mathrm{kg} / \mathrm{min}$ ute, and the doses of adrenaline and noradrenaline were increased to $1 \mu \mathrm{g} / \mathrm{kg} /$ minute. Erythrocyte suspension was infused when the hemoglobin value dropped to $2.8 \mathrm{gr} / \mathrm{dL}$, and thrombocyte suspension was transfused when the platelet count decreased to $22.000 / \mathrm{mm}^{3}$. In order to achieve the hemoglobin level at the target concentration of at least $10 \mathrm{gr} /$ $\mathrm{dL}, 4$ erythrocyte suspensions $(10 \mathrm{cc} / \mathrm{kg})$ at 6 -hour intervals were transfused over 12 hours. Since prothrombin time (PT) was 24 seconds and the international normalized ratio was 2.16 , IV fresh frozen plasma was administered. Whole blood count, PT, and partial thromboplastin time values were checked at 4-hour intervals, and supplementation with blood products was provided as needed. The procalcitonin level of the patient was $12.59 \mathrm{ng} / \mathrm{mL}$. Combined antibiotherapy with meropenem, vancomycin, and amikacin was maintained, and metronidazole was added to this regimen. Despite all efforts, his hypotensive state did not improve, so a single dose of IV steroid was administered, but was without any favorable outcome. A 3-F catheter was 
inserted into the femoral vein, and cardiac output was monitored using a $\mathrm{PiCCO}$ pulse contour monitor. Following thermodilution, measurements were as follows: cardiac index: $6.5 \mathrm{~L} /$ minute (normal range: 3-5 L/minute), systemic vascular resistance index (SVRI): 338 dynes $/$ second $/ \mathrm{cm}^{5} / \mathrm{m}^{2}$ (normal range: $1200-2000$ dynes $/$ second $/ \mathrm{cm}^{5} / \mathrm{m}^{2}$ ), global end-diastolic index (GEDI): $780 \mathrm{~mL} / \mathrm{m}^{2}$ (normal range: $680-800 \mathrm{~mL} / \mathrm{m} 2$ ), extravascular lung water index (EVLWI): $15 \mathrm{~mL} / \mathrm{kg}$ (normal: $<10 \mathrm{~mL} / \mathrm{kg}$ ), systolic blood pressure (SBP): $67 \mathrm{mmHg}$, diastolic blood pressure (DBP): $32 \mathrm{mmHg}$, mean arterial blood pressure (MAP): $46 \mathrm{mmHg}$, and a pulse rate of $106 /$ minute. Due to the low SVRI, TP was administered as an IV bolus dose of $10 \mu \mathrm{g} / \mathrm{kg}$. Since a life-threatening condition requiring emergency intervention existed, consent of the family could

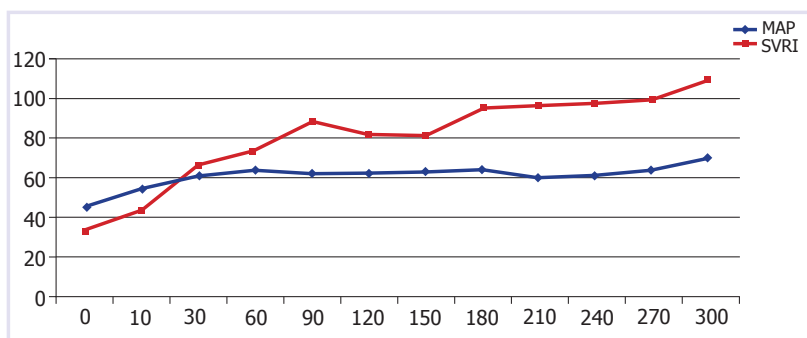

FIGURE 1. Ten minutes after a bolus dose of terlipressin, a marked increase in MAP and SVRI values were observed. The increases in MAP and SVRI were maintained with subsequent TP infusion.

MAP: Mean arterial blood pressure, $\mathrm{mmHg}$; SVRI: Systemic vascular resistance index, dynes $/ \mathrm{s} / \mathrm{cm}^{5} \mathrm{~m}^{2} / 10$.
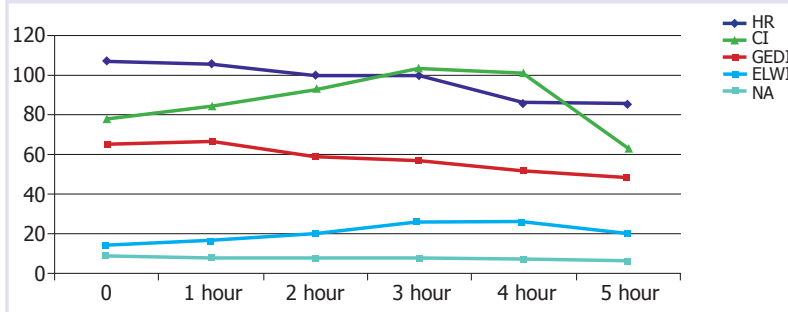

FIGURE2. One hour after intravenous bolus of terlipressin and subsequent infusion, a decrease was observed in $\mathrm{HR}$ and $\mathrm{CI}$, and an increase in GEDI and ELWI. After initiation of CVVH, GEDI and EVLWI decreased.

CVVH: Continuous venovenous hemodiafiltration; CI: Cardiac index, l/min/10; EVLWI: Extravascular lung water index;GEDI: Global end-diastolic index, x10; HR: Heart rate; NA. Noradrenaline, $(\mu \mathrm{g} / \mathrm{kg} / \mathrm{min}) \times 10$. not be obtained due to the time constraint, but consent was obtained when the patient was moved to intensive care. Ten minutes after the TP application, a marked increase in blood pressure was noticed. SBP, DBP, and MAP increased to $94 \mathrm{mmHg}$, $45 \mathrm{mmHg}$, and $60 \mathrm{mmHg}$, respectively. TP treatment was changed to infusion at a dose of $1 \mu \mathrm{g} / \mathrm{kg} /$ minute. The noradrenaline dose was reduced to 0.7 $\mu \mathrm{g} / \mathrm{kg} /$ minute, with decrements of $0.1 \mu \mathrm{g} / \mathrm{kg} / \mathrm{min}$ ute. A decrease in both the cardiac index and heart rate was observed (Fig. 1), while SVRI, GEDI, and EVLWI increased. Continuous venovenous hemodiafiltration (CVVH) was initiated, and $1000 \mathrm{cc}$ ultrafiltrate was aspirated over 12 hours. Control BUN and creatinine values were $27 \mathrm{mg} / \mathrm{dL}$ and 1.9 $\mathrm{mg} / \mathrm{dL}$, respectively. After initiation of CVVH, a decrease in GEDI, and ELWI values were detected (Fig. 2). At the ninth hour of TP infusion, ischemic manifestations developed on the right big toe, which necessitated the discontinuation of TP infusion. At approximately 1 hour after discontinuation of TP, SBP of $69 \mathrm{mmHg}$, DBP of $46 \mathrm{mmHg}$, and MAP of $54 \mathrm{mmHg}$ were measured. Three hours later cardiac arrest developed. Cardiopulmonary resuscitation was applied, but the patient was lost.

\section{DISCUSSION}

Sepsis and septic shock are important causes of morbidity and mortality in critically ill children. Septic shock is a form of distributive shock characterized by arterial and venous vasodilatation. Patients usually have a high cardiac index, and a lower systemic vascular index. In sepsis, inflammatory cytokines are secreted from the vascular endothelium, neutrophils, central nervous system, and neuroendocrine system, and these mediators stimulate nitric oxide (NO) activity. ATP-sensitive potassium channels are activated by the NO, and the passage of calcium slows down. The vascular response to catecholamines decreases, and nearly $40 \%$ of patients are lost to treatment-refractory shock, despite supportive treatment $[2,3]$. In treatment, achieving mean arterial blood pressure to maintain adequate organ perfusion is targeted; however, the development of vascular hyporeactivity against cat- 
echolamines has led to the search for an alternative vasopressor agent.

Vasopressin is a known antidiuretic hormone that is synthesized from the hypothalamus. Its effects are seen in the vascular V1a, and renotubular $\mathrm{V} 2$ receptors. The $\mathrm{V} 1$ a receptors on vascular smooth muscle induce increases in the intracellular concentration of calcium, which leads to arterial vasoconstriction. Under stimulation of V2, renal water reabsorption increases. Its half-life is only 6 minutes, and its effects are seen for 30 to 60 minutes. TP is a synthetic triglycyl lysine analogue of vasopressin, and a precursor of lypressin. It is a long-acting (2-10 hours) analogue of vasopressin compared with both lypressin and vasopressin. Its half-life is 6 hours, and it is a more potent vasoconstrictor agent than vasopressin. Its half-life enables treatment with bolus doses. Since vasopressin has a half-life of only 6 minutes, it must be given as an infusion. The recommended infusion doses of vasopressin and TP are $0.03 \mathrm{U} /$ minute, and $1.3 \mathrm{mcg} / \mathrm{kg} /$ hour, respectively [7]. In patients with septic shock, withdrawal of a vasopressin infusion may induce the phenomenon of "rebound" hypotension $[4,5]$.

Landry et al. [6] first reported the beneficial effects of vasopressin in patients with septic shock when adequate blood pressure could not be maintained despite inotropic and vasoconstrictor treatment.

In the Continuous Terlipressin Versus Vasopressin Infusion in Septic Shock (TERLIVAP) study, 45 patients with septic shock were randomized into TP $(1.3 \mu \mathrm{g} / \mathrm{kg} /$ hour $)$, vasopressin $(0.03 \mathrm{U} /$ minute $)$ and titrated noradrenaline groups, and no conspicuous hemodynamic intergroup difference was detected. However, the reduced risk of adverse effects associated with a continuous infusion of TP over bolus doses was emphasized [7].

Morelli et al. [8] reported increases in MAP, gastric mucosal perfusion, urine output, and creatinine clearance in 15 patients with septic shock whose MAP values ranged between $50-55 \mathrm{mmHg}$ following administration of an IV bolus dose of TP. TP increases perfusion pressure and the glomerular filtration rate with a vasodilatory effect on efferent arterioles.
The results of a study of adult patients indicated a decrease in oxygenation index (OI) immediately after administration of TP, though it was not statistically significant. As reported by the researchers, the decrease in OI was potentially associated with vasodilation induced by the impact of TP on pulmonary blood vessels [9].

In a multicenter study performed in Spain in 2006, bolus doses of TP were administered every 4 hours, and a rapid increase in MAP necessitated tapering the catecholamine infusion 1 hour after the bolus dose [10].

Yildızdaş et al. [11] evaluated the effect of TP in 58 septic shock patients with persistent hypotension despite fluid therapy and high doses of catecholamines. They administered IV bolus doses of $20 \mu \mathrm{g} / \mathrm{kg}$ TP, and after 30 minutes, MAP and $\mathrm{PaO}_{2} /$ $\mathrm{FiO}_{2}$ markedly increased, and the heart rate significantly decreased. However, they indicated a lack of any difference between TP and control groups . In a similar study performed by Albenése et al. [12], the researchers demonstrated that when they randomized hyperdynamic septic shock patients into noradrenaline and bolus TP groups, both drugs increased MAP and decreased heart rate, $\mathrm{Cl}$, and $\mathrm{O}_{2}$ consumption.

PiCCO data of the effects of TP in septic shock patients have been evaluated only in case reports $[13,14]$. Peters et al. [13] administered bolus TP to an 11-year-old patient with septic shock whose hypotension persisted despite fluid support, dopamine, and noradrenaline infusions. After each bolus of TP, there was a rapid increase in the SVRI, and the noradrenaline dose could be tapered within approximately 6 hours, and then discontinued. In our case as well, 10 minutes after $10 \mu \mathrm{g} / \mathrm{kg}$ bolus TP, the SVRI markedly increased, and 5 hours later, the noradrenaline dose could be reduced from $1 \mu \mathrm{g} / \mathrm{kg} /$ minute to $0.7 \mu \mathrm{g} / \mathrm{kg} /$ minute.

Excess vasoconstriction and impairment of tissue perfusion are unwanted potential effects of vasopressin and TP. Ischemic effects were observed on the right big toe of our patient, and so the TP infusion was terminated.

TP may be a treatment alternative in catechol- 
amine-resistant septic shock patients; however, additional randomized controlled studies are needed to fully provide an answer on its use.

Conflict of Interest: No conflict of interest was declared by the authors.

Financial Disclosure: The authors declared that this study has received no financial support.

Authorship contributions: Concept - M.B., S.E.; Design S.E.; Data Collection - M.B.,S.E.; Analysis - M.B.; Literature search - S.E.; Writing - S.E.

\section{REFERENCES}

1. De Backer D, Biston P, Devriendt J, Madl C, Chochrad D, Aldecoa $\mathrm{C}$, et al. Comparison of dopamine and norepinephrine in the treatment of shock. N Engl J Med 2010;362:779-89. [CrossRef]

2. Watson RS, Carcillo JA, Linde-Zwirble WT, Clermont G, Lidicker J, Angus DC. The epidemiology of severe sepsis in children in the United States. Am J Respir Crit Care Med 2003;167:695-701. [CrossRef]

3. Landry DW, Oliver JA. The pathogenesis of vasodilatory shock. N Engl J Med 2001;45:588-95. [CrossRef]

4. Asfar P. Terlipressin in chronic hyperdynamic endotoxic shock: is it safe? Intensive Care Med 2003;29:154-5. [CrossRef]

5. Nilsson G, Lindblom P, Ohlin M, Berling R, Vernersson E. Pharmacokinetics of terlipressin after single i.v. doses to healthy volunteers. Drugs Exp Clin Res 1990;16:307-14.
6. Landry DW, Levin H, Gallant E, Seo S, D'Alessandro D, Oz $\mathrm{MC}$, et al. Vasopressin pressor hypersensitivity in vasodilatory septic shock. Crit Care Med 1997;25:1279-82. [CrossRef]

7. Morelli A, Ertmer C, Rehberg S, Lange M, Orecchioni A, Cecchini $\mathrm{V}$, et al. Continuous terlipressin versus vasopressin infusion in septic shock (TERLIVAP): A randomized, controlled pilot study. Crit Care 2009;13:130. [CrossRef]

8. Morelli A, Rocco M, Conti G, Orecchioni A, De Gaetano A, Cortese $\mathrm{G}$, et al. Effects of terlipressin on systemic and regional haemodynamics in catecholamine-treated hyperkinetic septic shock. Intensive Care Med 2004;30:597-604. [CrossRef]

9. Matok I, Vard A, Efrati O, Rubinshtein M, Vishne T, Leibovitch $\mathrm{L}$, et al. Terlipressin as rescue therapy for intractable hypotension due to septic shock in children. Shock 2005;23:305-10. [CrossRef]

10. Nunez AR, Lopez HJ, GilAJ, Hernandez A, Rey C. REDSPET Working Group of the Spain Society of Pediatric Intensive Care. Crit Care 2006;10:1-8. [CrossRef]

11. Yildızdaş D, Yapıcıoğlu H, Çelik Ü, Sertdemir Y, Alhan E. Terlipressin as a rescue therapy for catecholamine-resistant septic shock in children. Intensive Care Med 2008;34:511-7. [CrossRef]

12. Albanese J, Leone M, Delmas A, Martin C. Terlipressin or norepinephrine in hyperdynamic septic shock: a prospective, randomized study. Crit Care Med 2005;33:1897-902. [CrossRef]

13. Peters MJ, Booth AB, Petros AJ. Terlipressin bolus induces systemic vasoconstriction in septic shock. Pediatr Crit Care Med 2004;5:112-5. [CrossRef]

14. Holley DH, De Keulenaer BL, Potter A, Stephens DP. Terlipressin infusion in catecholamine-resistant shock. Anaesth Intensive Care 2003;31:560-4. 